\title{
Ultra-high frequency sonochemistry and its dosimetry
}

\author{
Xiangjun Wang ${ }^{1,2}$, Jun Gao $^{2}$ \& Ping zhang ${ }^{1,2}$ \& Ronghui Lin $^{1,2}$ \& Yixin Yang ${ }^{1,2}$ \& \\ Hui You $^{2^{*}} \&$ Xiaofei Wang ${ }^{2}$
}

${ }^{1}$ Department of Automation, University of Science and Technology of China, Hefei 230026, China

${ }^{2}$ Institute of Intelligent Machines, Chinese Academy of Science, Hefei230031, China

Keywords: the ultrasonic cavitation effect, iodie dosimetry, conductivity dosimetry, fluorescence dosimetry

\begin{abstract}
MHz}$ and $2.4 \mathrm{MHz}$ focused ultrasonic transducer were chosen in this article as ultrasonic source. Three methods were used to characterize the ultrasonic cavitation effect. They are iodine dosimetry, conductivity dosimetry, fluorescence dosimetry. The acoustic energy radiation in the liquid was evaluated using calorimetry. The influence of ultrasound intensity and ultrasonic treatment time was analysed. The correlation between sonochemical efficiency and corresponding cavitation yield in terms of energy was studied. Through iodide dosimetry and fluorescence dosimetry, we obtained the relationship between the cavitation effect in solution and the solution concentration.
\end{abstract}

\section{Introduction}

In the 80s of last century, sonochemistry have begun to emerge as a new field of intersectional subject, which gets more attention from both the scientific community and the industrial circles[1]. Now, applications of ultrasound wave technology include synthesis of pharmaceuticals, products, foodstuffs , advanced treatment of waste water, therapy and diagnosis[2-6]. Due to its wide application in many fields, ultrasonic wave has attracted great interest and has been extensively studied. Acoustic cavitation is the nonlinear effect resulting from the interaction between sound and fluid medium[7]. The effects of ultrasound originate primarily in acoustic cavitation. Ultrasound includes two big aspects, one is high-frequency ultrasound (mainly ranging from $1 \mathrm{MHz}$ to $10 \mathrm{MHz}$ ), the other is power ultrasound for the purpose of process intensification (mainly ranging from $20 \mathrm{KHz}$ to $50 \mathrm{KHz}$ ). Hitherto, most of sonochemical research use 20 to $50 \mathrm{KHz}$ low-frequency as ultrasonic radiation source. The investigation of the mechanics of ultrasonic cavitation effect is rare at $\mathrm{MHz}$ level.

Due to the ultrasonic cavitation occurred in extreme conditions and the process is transient, the direct detection of acoustic is quite difficult. The study on ultrasonic cavitation are usually based on some different effect produced by ultrasonic cavitation. There effects are the generation of heat, mechanical effects, optical effects and chemical effects[8]. In our paper, by detecting chemical effects produced by ultrasonic cavitation, we can determine the cavitation yields. The chemical dosimetries are based on oxidation or reduction reactions occurring in an aqueous solution[9]. In order to help us to know more about the relationship between the efficiency of sonochemical reaction and the energy of ultrasonic irradiation used to produce them, the ultrasonic power should be attained. Due to the fact that energy conversion of ultrasound varies with each transducer, the input electric power is not always informative to ultrasonic power for sonochemistry. The acoustic power dissipated into solutions was determined by calorimetric method using liquid. Such a calibration cannot distinguish between the waste heat and the acoustic-phase heat[10]. Calorimetry is based on the assumption that almost all the mechanical energy produces heat and the output power can be obtained.

Chemical dosimetry gives sonochemical efficiency in a whole reaction solution. In this paper, we report the chemical effects of sonochemical reaction investigated by means of three dosimetries(iodine dosimetry, conductivity dosimetry, fluorescence dosimetry). Another objective is to obtain a fundamental understanding of sonochemical oxidation by varying initial sample 
concentration. Further, we analyzed sonochemical efficiency at two frequencies $2 \mathrm{MHz}$ and $2.4 \mathrm{MHz}$.

\section{Experimental}

\section{Sonochemical reactor}

A schematic experimental setup is shown in Fig.1.The sonochemical vessel was made of a cylindrical glass tube with thickness and inner diameter of 3 and 23mm, respectively. The height of the vessel was $150 \mathrm{~mm}$. The sonochemical reactor without a jacket was placed at the room temperature. A transducer was mounted below the glass tube as shown in the picture. The detailed specifications of transducers are listed in Table 1. In this paper, the irradiation frequencies were 2.0MHz and $2.4 \mathrm{MHz}$. The transducers were driven by a low-frequency power amplifier.

\begin{tabular}{|c|c|c|c|c|}
\hline No. & $\begin{array}{l}\text { Type of } \\
\text { transducer }\end{array}$ & $\begin{array}{l}\text { Drive } \\
\text { frequency(MHz) }\end{array}$ & $\begin{array}{l}\text { Drive } \\
\text { electric } \\
\text { power(W) }\end{array}$ & $\begin{array}{l}\text { Ultrasound } \\
\text { power(W) }\end{array}$ \\
\hline 1 & WHQ2018 & 2.0 & $36 \mathrm{~W}$ & 3.78 \\
\hline 2 & WHQ2418 & 2.4 & $24 \mathrm{~W}$ & 2.65 \\
\hline
\end{tabular}

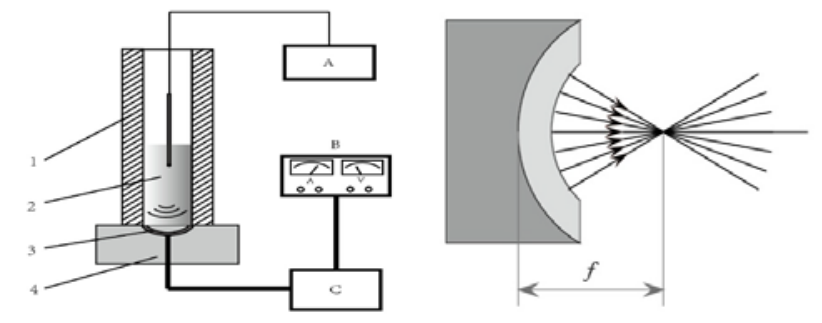

Fig.1.a Experimental setup: 1:organic class tube;2:sample

solution;3:Transducer;4:polydimethylsiloxane;A:Thermometer;B:single generator; C:Power amplifier. Fig.1.b the principle diagram of the spherical focusing transducer. ' $\mathrm{f}$ ' is the focal length.

\section{Power measurement and dosimetry}

When the ultrasonic propagates in liquid media, due to the ultrasonic radiation effect, the temperature of high sound absorption material rises. By measuring the initial rate of the temperature rise, ultrasonic power or sound intensity can be measured. The ultrasonic power dissipated into the solution was measured with a standard calorimetry by using the following relation:

$$
\text { Power }=\left(\frac{d T}{d t}\right) C_{p} M
$$

Where $\mathrm{m}$ is the mass of liquid, $C_{p}$ is the heat capacity of the liquid and $(d T / d t)$ is the initial slope of the curve of temperature versus time. For the system under study the initial temperature rise (T) was recorded against time (t) for about 60s, using a thermocouple placed in the reaction vessel. Using the above equation, the ultrasonic power radiated into the liquid is calculated as $3.78 \mathrm{~W}$ and 2.65W, respectively. The power efficiency obtained by dividing the ultrasonic power dissipation into solution by the input electric power was $10.5 \%$ at $2 \mathrm{MHz}$ at $36 \mathrm{~W}$ input and $11 \%$ at $2.4 \mathrm{MHz}$ at $24 \mathrm{~W}$ input. Thermally determined power was independent of the initial environment temperature $(0$ to 40C) at several dial setting power levels.

\section{Sonochemical efficiency}

The sonochemical efficiency (SE), defined as the ratio of the number of reacted molecules to the ultrasound energy, is given by

$$
S E=\frac{C V}{P t}
$$

Where $C$ is the concentration of the $\mathrm{I}_{3}^{-}$ion of the KI solution or $\mathrm{H}_{2} \mathrm{O}_{2}$ formed and $V$ is the solution volume. $P(\mathrm{w})$ is the calorimetrically determined power. 


\section{Result and discussion}

\section{Quatification of sonochemical reaction}

\section{1) Iodide dosimetry}

In Iodide dosimeter, we use $0.2 \mathrm{~mol} / \mathrm{L}, 0.6 \mathrm{~mol} / \mathrm{L}, 1.5 \mathrm{~mol} / \mathrm{L}$ concentration solutions as ultrasonic sample. The result cavitation yield versus ultrasonic time have been presented in the form of a plot as shown in Fig.2. From the figure, it can be seen that with the greater initial solution concentration, the slope is also higher. At the same time, As the time increase, cavitation yield increased accordingly. Although the time course deviate slightly from the linearity at longer irradiation time, the product yields and the decomposition ratio on sonication reflect the sonochemical effect. The sonochemical efficiency is evaluated by the concentration of the compounds divided by the ultrasonic energy density. In other words, the sonochemical efficiency is the ratio of the number of reacted molecule toward the ultrasonic energy. The plot of the sonochemical efficiency against ultrasonic as shown in Fig.3. The value of sonochemical efficiency become decline, at longer irradiation time. When the ultrasonic power of $1 \mathrm{~W}$ for $1 \mathrm{~s}$ is dissipated into the liquid, the cavitation yield gets smaller at longer ultrasonic treatment time.
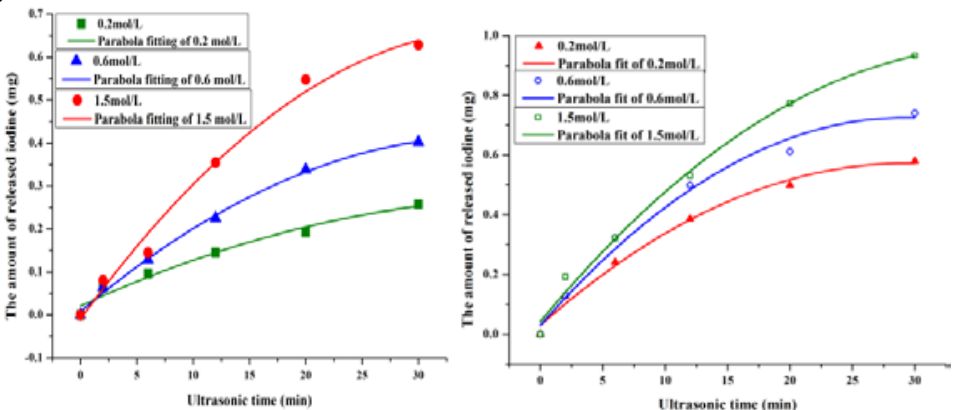

Fig.2. plot of cavitation yield of different initial concentration of solution against ultrasonic time. Fig.2.a ultrasonic frequency is $2 \mathrm{Mhz}$.Fig2.b ultrasonic frequency is $2.4 \mathrm{MHz}$.
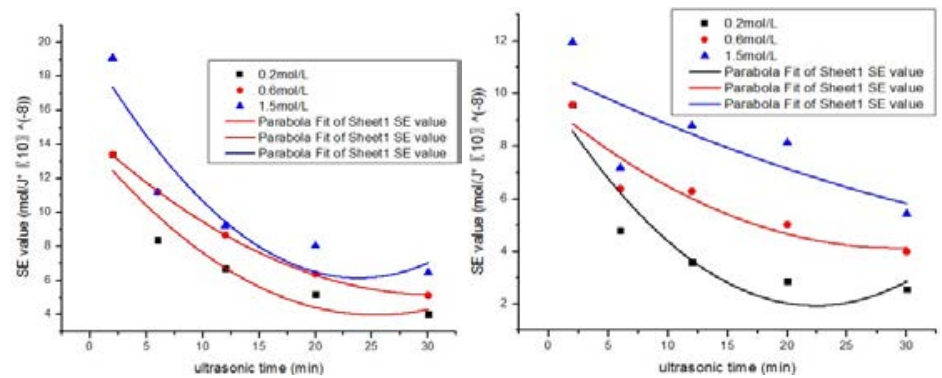

Fig.3 .Plot of SE (the sonochemical efficiency) value against ultrasonic time. For Fig.3.a Ultrasonic treatment frequency is $2 \mathrm{MHz}$ and Fig.3.b ultrasonic treatment frequency is $2.4 \mathrm{MHz}$.

\section{2) Conductivity dosimetry}

In this experiment, we chose deionized water as ultrasonic samples at room temperature. The cavitation yield size was judged by the change of the conductivity. The result is shown in the figure below. In our experiment, the electric conductivity of the sample was measured just before and after sonication with a electric conductivity meter. From the Fig.4, there is a linear relationship between the time of ultrasonic treatment and the ultrasonic cavitation yield. At longer irradiation time, the time course slightly from the linearity. 


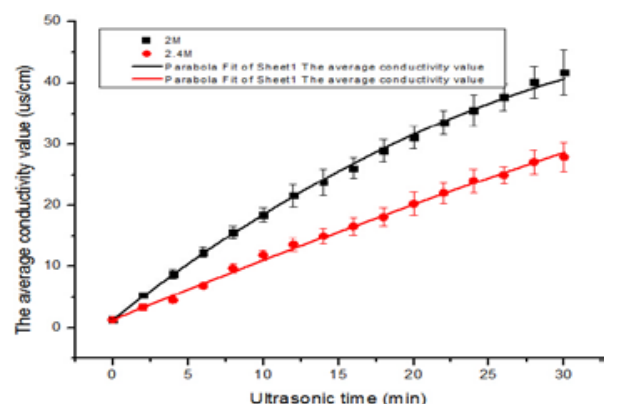

Fig.4.plot of the average conductivity value against ultrasonic time. The average is the average of the five times the

\section{3) fluorescence dosimetry} result of the experiment.

In the alkaline aqueous solution, terephthalic acid produces terephthalate anions that react with hydroxyl radicals to generate highly fluorescent 2-hydroxyterephthalate ions (HTA). In this dosimetry, four kinds of concentration of solution could be chosen fluorescence liquid sample that were respectively $0.5 * 10^{-3} \mathrm{~mol} / \mathrm{L}, 1.0^{*} 10^{-3} \mathrm{~mol} / \mathrm{L}, 1.5^{*} 10^{-3} \mathrm{~mol} / \mathrm{L}, 2.0^{*} 10^{-3} \mathrm{~mol} / \mathrm{L}$. Detection of 2-hydroxyterephthalate was by fluorimetry; $\lambda$ (excitation) $=310 \mathrm{~nm} ; \lambda$ (emission) $=425 \mathrm{~nm}$, using authentic reference material. Through the detection of the change of absorbance terephtalic acid salt ions at $310 \mathrm{~nm}$, hydroxyl free radicals generated by ultrasonic can be quantitative detected to some extent. The result is shown in Fig. 5 below. When the ultrasonic treatment time is less than 20min, there is a linear relationship between the time of ultrasonic treatment and the ultrasonic cavitation yield.
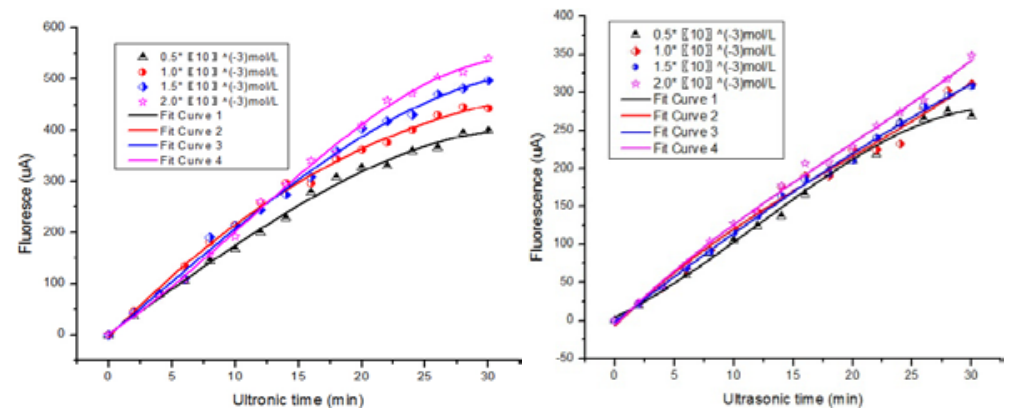

Fig.5 plot of different initial concentration solution against ultrasonic time.Fig.5.a ultrasonic treatment frequency is 2MHz. Fig.5.b ultrasonic treatment frequency is $2.4 \mathrm{MHz}$.

\section{Conclusions}

The relationship of sonochemical and the ultrasonic power was investigated using HIFU transducers at $2 \mathrm{MHz}$ and $2.4 \mathrm{MHz}$. It found that there are linear relationship between the ultrasonic treatment time and cavitation yield. Zhaohua Chen et al[11] that the sonochemical yield varies linearly with sonication time. With the greater initial solution concentration, the rate of ultrasonic cavitation reaction is also higher. Since the sound pressure field distribution is not uniform the result we attained is the average. As the Fig4 shows, the slopes of the two straight lines is about 1.62, while the ultrasonic power determined by calorimetrical is about 1.43 . The two difference between the two numbers is small. In fluorescence dosimetry, as shown in the two picture in Fig.6. The slope ratio corresponding to the same initial concentration of ultrasonic solution are about the same. The value of this straight slope and the value of the ratio of two frequency corresponding ultrasonic power dissipated into the liquid are approximately equal. We believe that approximation values of the two are equal. The SE values obtained from Eq.2 are plotted against the ultrasonic treatment time in Fig.3. It was found that with the ultrasonic treatment time get longer, the SE values decrease. From Fig.3 ,it was found that the ratio of two frequency corresponding to the SE values are about equal in size. The result of the three dosimetries jointly reflect the fact that these values are a measure of sonochemical effect. The sonochemical efficiency has nothing to do with the frequency if the output power of the ultrasonic transducer are the same. 


\section{Acknowledgement}

This work is supported by National Natural Science Foundation of China (No. 61176105), Hundred Talents Program of Chinese Academy of Science and Found of President of the Chinese Academy of Sciences( YZJJ201316). Hui You is co-corresponding author of this paper and his contact is hyou@iim.ac.cn.

\section{References}

[1] JIA Wei-gao, ZHANG Peng, LIU Zhen-rorg, LI Hui-fang, YANG Qi, WANG Jun, A research and An Application of the Sonochemistry, Journal of Liaoning University Natural Sciences Edition, 29(3),pp.198-201, 2002.

[2] Adewuyi, Sonochemistry in Envrionmental Remediation.1.Combinative and hybrid sonophotochemical oxidation processes for the treatment of pollutants in water,Environmental Science \& Technology,39(10),pp.3409-3420, May 2005.

[3] Gonzalez-Garcia, J; Saez,V; Tudela, I; Diez-Garcia, MI; Esclapez, MD; Louisnard, O. Sonochemical Treatment of Water Polluted by Chorinated organocompounds, Water, 2(1),pp.2874 ,Mar 2010.

[4] KE Zhang,Bong-Jun Park,Fei-fei Fang,Hyoung Jin Choi*, Sonochemical Preparation pf Polymer Nanocomposites,Molecules,14(6),pp.2095-2110, Jun 2009.

[5] Shchukin,Dmitry G; Radiuk, Darya; Moehwald, Helmuth. Ultrasonic Fabrication of Metallic Nanomaterical and Nanoalloys, Annual review of Materials research, 40,pp.345-362, 2010.

[6] Fang,Nicholas;Xi,Dongjuan;Xu,Jianyi;ultrasonic metamaterials with negative modulus, Natural materials,5(6),pp.452-456,Jun 2006

[7] YUAN Jian-gang, YAN Bi-ge, YANG Yan-ni, Review on Measurement of Ultrasound Cavitation Intensity, Progress in Modern Biomedicine,7(2),pp.295-297,Oct 2007.

[8] Yufei Hu, Zhujun Zhang*,Chunyan Yang, Measurement of hydroxyl radical production in ultrasonic aqueous solutions by a novel chemiluminescence method, Ultrasonics Sonochemistry ,15(5),pp.665-672,J ul 2008.

[9] Y. Iida*, K. Yasui, T. Tuziuti, M. Sivakumar, sonochemistry and its dosimetry ,Microchemical Journal ,80(2),pp.159-164, Jun 2005.

[10] Shinobu Koda*,Takahide Kimura, Takashi Kondo, Hideto Mitome, A Standard method to calibrate sonochemical efficiency of an individual reaction system ,ultrasonic sonochemistry, 10(3),pp.149-156, May 2003.

[11] CHEN Zhaohua ,ZHU Changping, HAO Yiyun WANG Hui ,FENG Ruo, Study on the sonochemical yield of low frequency ultrasound by iodine release method, Acoustic Technology,16(4),pp.192-197, Oct 1997. 